\title{
Factors determining self-assembly of hyaluronan
}

\author{
Karolína Kocourková $^{\mathrm{a}, \mathrm{b}}$, Lenka Musilová ${ }^{\mathrm{a}, \mathrm{b}}$, Petr Smolka ${ }^{\mathrm{a}, \mathrm{b}}$, Aleš Mráček ${ }^{\mathrm{a}, \mathrm{b}}$, Martin Humenik ${ }^{\mathrm{c}}$, \\ Antonín Minařík ${ }^{\mathrm{a}, \mathrm{b}, *}$ \\ ${ }^{a}$ Department of Physics and Materials Engineering, Tomas Bata University in Zlín, Vavrečkova 275, 760 01, Zlín, Czech Republic \\ ${ }^{\mathrm{b}}$ Centre of Polymer Systems, Tomas Bata University in Zlín, Trúda Tomáš Bati 5678, 760 01, Zlín, Czech Republic \\ ${ }^{\mathrm{c}}$ Department of Biomaterials, Faculty of Engineering Science, Universität Bayreuth, Prof.-Rüdiger-Bormann. Str. 1, 95447, Bayreuth, Germany
}

\section{A R T I C L E I N F O}

\section{Keywords:}

Hyaluronan

Atomic force microscopy

Polysaccharides

Network

Aggregates

\begin{abstract}
A B S T R A C T
The conditions determining network-forming and aggregation properties of hyaluronan on the mica surface were studied. The hyaluronan was deposited on the surface from aqueous and saline solutions and attached by a bivalent cation. The morphology of the immobilized assemblies was characterized by atomic force microscopy. The experimental results show that the morphology and size of the aggregates as well as the density of the interconnecting fibrillar network, both made of hyaluronan, at the liquid-solid phase interface are determined not only by its molecular weight or concentration in solution, but also by the dissolution conditions and storage time. These findings extend the current state of knowledge about the conformational variability of this biologically important polymer. Understanding the conformational variability is of great importance, as it governs the physiological functions of hyaluronan, as well as its processability and formulations. That in turn determines its usability in different pharmacological and biomaterial applications.
\end{abstract}

\section{Introduction}

Hyaluronic acid, or rather its sodium salt - hyaluronan (HU), has a number of important biological functions, which are closely related to its molecular weight, concentration and spatial arrangement of the polymer chains (Dicker et al., 2014; Evanko, Parks, \& Wight, 2016). Due to its natural occurrence in the human body, it is used in a wide range of pharmaceutical and biomedical applications (Dosio, Arpicco, Stella, \& Fattal, 2016; Highley, Prestwich, \& Burdick, 2016). Despite the considerable amount of work devoted to this polysaccharide, new studies are constantly expanding the knowledge-base on this macromolecular system (Fallacara, Baldini, Manfredini, \& Vertuani, 2018; Grossutti \& Dutcher, 2016; Mondschein, Kanitkar, Williams, Verbridge, \& Long, 2017).

The specific properties and bioactivity of $\mathrm{HU}$ are closely related to the spatial arrangement of the macromolecule. The conformational configuration of $\mathrm{HU}$ in solution varies radically over a wide range of concentrations from a random coil form with a significant degree of flexibility to semi-organized polymer networks (Day \& Sheehan, 2001). These networks are highly complex in a biological environment (Heinegård, 2009). They affect the transport of water, limit the movement of pathogens (Xu, Jha, Harrington, Farach-Carson, \& Jia, 2012), efficiently absorb vibrations in extracellular environment, effectively control the diffusion of proteins (Dicker et al., 2014) and are among the components responsible for the mechanical properties of soft tissues (Scott, Cummings, Brass, \& Chen, 1991). The formation and stability of the network structure in tissues is determined by high concentration of HU. For example, human vitreous humor contains $\mathrm{HU}$ of molecular weight 2-4 $\times 10^{6} \mathrm{Da}$ in concentration $65-400 \mu \mathrm{g} / \mathrm{mL}$ (Bishop, 2000).

In contrast, HU networks under in vitro conditions are much simpler. They consist of randomly interconnected HU fibres with unstable mechanical properties (Scott \& Heatley, 1999; Xu et al., 2012). The impact of this can be observed, for example, in the treatment of osteoarthritis by injecting $\mathrm{HU}$ as lubricant, where the viscosity of the HU solution decreases dramatically under shear stress. This problem can be overcome by chemical treatment or formulations leading to morphological changes of the HU networks (Zheng et al., 2019). It is known that the preparation of HU-based hydrogels and scaffolds under in vitro conditions very often depends on the functionalization of polymer chains (Deng et al., 2017; Foglarová et al., 2016; Nimmo, Owen, \& Shoichet, 2011; Van Vlierberghe, Dubruel, \& Schacht, 2011).

In diluted solutions, $\mathrm{HU}$ chains occur in the form of random coils that form a temporary polymer network, while at high concentrations the network density increases and the macromolecules cluster into

\footnotetext{
* Corresponding author at: Department of Physics and Materials Engineering, Tomas Bata University in Zlín, Vavrečkova 275, 760 01, Zlín, Czech Republic.

E-mail address: minarik@utb.cz (A. Minařík).
} 
aggregates (Lapcík, Lapcík, De Smedt, Demeester, \& Chabrecek, 1998; Matteini et al., 2009; Scott et al., 1991). The driving forces of aggregation are hydrophobic interactions and hydrogen bonds compensating the electrostatic resistance between polyanionic charges (Gribbon, Heng, \& Hardingham, 1999; Rinaudo, 2006; Scott, 1992). Further, the properties of the polymer network are strongly dependent on the molecular weight of HU (Shen, Chaudouet, Ji, \& Picart, 2011; Wieland et al., 2016). Longer chains show more extensive interweaving and higher number of interchain interactions. The resulting network is therefore more coherent and larger (Scott et al., 1991). The formation of HU network and the conformation changes are also conditioned by the $\mathrm{pH}$ of the solution and by the concentration, type and valence of the ions in the solution (Giubertoni et al., 2019; Gŕundělová, Mráček, Kašpárková, Minař́́k, \& Smolka, 2013; Mráček et al., 2015; Zellermann, Bergmann, \& Mayer, 2013).

HU networks properties have already been analyzed by various techniques, e.g. rotary shadowing electron microscopy (Scott et al., 1991), X-ray crystallography (Hargittai \& Hargittai, 2008), NMR spectroscopy (Almond, DeAngelis, \& Blundell, 2006; Cowman, Hittner, \& Feder-Davis, 1996; Nestor \& Sandström, 2017; Scott \& Heatley, 1999), light scattering (Kim, Woo, Park, Hwang, \& Moon, 2015), tensiometry (Musilová, Kašpárková, Mráček, Minařík, \& Minařík, 2019), chromatography (Shanmuga Doss, Bhatt, \& Jayaraman, 2017), molecular dynamics (Cowman \& Matsuoka, 2005; Hargittai \& Hargittai, 2008; Kutálková, Hrnčiř́ík, Witasek, \& Ingr, 2020) and atomic force microscopy (AFM) (Cowman, Li, \& Balazs, 1998, 2005; Giannotti, Rinaudo, \& Vancso, 2007; Gunning, Morris, Al-Assaf, \& Phillips, 1996; Jacoboni, Valdrè, Mori, Quaglino, \& Pasquali-Ronchetti, 1999; Spagnoli et al., 2005; Zellermann et al., 2013). It has been proven, that despite the effect of the surface and adsorbed water described by Cowman et al. (2005), AFM can be used to study the aggregation and networking ability of HU with respect to the process conditions of solution preparation. For that reason, the AFM microscopy has been chosen as a dominant experimental technique in this work.

Herein, we show that the self-assembly of $\mathrm{HU}$ is controlled by the combination of chemical factors such as molecular weight $(M W)$ and concentration of the biopolymer with processing factors such as temperature, dissolution method and storage time of the polymer solution. All these variables affect the physiologically important characteristics of the biopolymer system. The present study aims at establishing the link between processing conditions of solution preparation and aggregation behavior on interfaces, which is required for understanding the HUbased material properties such as coatings and films.

\section{Materials and methods}

\subsection{Chemicals}

Bacterial sodium salt of hyaluronic acid of various molecular weight in pharmaceutical purity was purchased from Contipro a.s. (Czech Republic). The weight average molecular weight $\left(M_{w}\right)$ of $\mathrm{HU}$ was in a range from $\sim 190 \mathrm{kDa}$ to $\sim 1000 \mathrm{kDa}$ as characterized by the AF4-MALS chromatography. As the dissolving media, $0.9 \%$ sodium chloride $(\mathrm{NaCl})$ solution (physiological solutions) acquired from Sigma-Aldrich and the ultra-pure water with the resistivity of $18.2 \mathrm{M} \Omega \cdot \mathrm{cm}$ were used. Magnesium chloride $\left(\mathrm{MgCl}_{2}\right)$ for the mica surface modification was also purchased from Sigma-Aldrich, as well as Tris/HCl buffer. Mica, serving as a substrate for HU to AFM imaging, was obtained from SPI SUPPPLIES in the V-4 quality.

\subsection{Molecular weight analysis}

The asymmetrical flow field-flow fractionation (AF4) was performed using the large channel (LC model) with trapezoidal design from Wyatt Technology Europe GmbH (Dernbach, Germany) equipped with the regenerated cellulose membrane (MWCO $30 \mathrm{kDa}$ ) from Merck Millipore
(Darmstadt, Germany) and $350 \mu \mathrm{m}$ spacer.

The HU samples were dissolved in $50 \mathrm{mM}$ Tris/ $\mathrm{HCl}$ buffer, $\mathrm{pH} 8.0$ (the carrier solution) at $1 \mathrm{mg} / \mathrm{mL}$ at RT for $16 \mathrm{~h}$. The injection volume was $180 \mu \mathrm{L}$ for each sample. The carrier solution was filtered through a nitrocellulose filter (pore size $=0.2 \mu \mathrm{m}$ ) from Millipore. After that, it was delivered by an Agilent 1100 Series HPLC system (Agilent, Germany). The system was additionally connected to a multi-angle light scattering detector DAWN EOS (Wyatt, Germany) and a refractive index detector Shodex RI-71 (Shodex, Germany). Molecular masses and root mean square (rms) radii from light scattering signals were calculated using the ASTRA 6 software (Wyatt, Germany).

\subsection{Solution preparation}

HU aqueous or physiological solutions with the concentration of 0.5 wt.\% were prepared by different dissolving ways of powder HU. The first type of solution was prepared on a laboratory orbital shaker at room temperature for 24 or $48 \mathrm{~h}$. The second way was realized on a magnetic stirrer at $45^{\circ} \mathrm{C}$ for $24 \mathrm{~h}$ and the last type of process involved dissolving in a temperature field flow chamber (TFFC) in temperature gradient for 24 h. Temperature gradient was generated by the temperature difference between the top cooling plate with $20{ }^{\circ} \mathrm{C}$ and the bottom heating plate with $50{ }^{\circ} \mathrm{C}$, as described in our previous works (Minařík et al., 2017). These solutions were stored at $4{ }^{\circ} \mathrm{C}$ and diluted to the concentration of 0.1 wt. $\%$ or 0.01 wt. \% prior to the preparation of the sample for AFM.

\subsection{Atomic force microscopy}

HU macromolecules deposited on the mica surface were imaged by the AFM. The freshly cleaved mica of area $1 \mathrm{~cm}^{2}$ was treated by $0.1 \mathrm{M}$ $\mathrm{MgCl}_{2}$. This solution was deposited on the mica surface in volume of 50 $\mu \mathrm{L}$ and dried by air flow after $60 \mathrm{~s}$ of incubation at room temperature. Then, $50 \mu \mathrm{L}$ of 0.1 or 0.01 wt. \% HU solution was deposited on the dry modified mica surface and incubated for $120 \mathrm{~s}$ at room temperature. The excess solution was then gently dried by air flow.

Unless otherwise stated, all process parameters for the deposition of the solution on the mica surface were kept constant. The samples were analyzed with the NTEGRA Prima (NT-MDT) microscope in the tapping mode in the air atmosphere. The silicon probe of force constant (1.45-15.1) N/m, type NSG01 (TipsNano) was used. The sample area was scanned in rate of $0.7 \mathrm{~Hz}$ in $512 \times 512$ pixel format.

\subsection{Image analysis}

The images were processed using the Gwyddion - Free SPM data analysis software, version 2.55 (D. Nečas, P. Klapetek, Czech Metrology Institute, Czech Republic). The area coverage was analyzed by the ImageJ software, version 1.5 (W. Rasband, National Institutes of Health, United States), the aggregates and network surface covering was obtained and averaged from 5 areas, $1 \mu \mathrm{m}^{2}$ each. Herein, the image analysis is presented for the most significant statistical data. Complete numerical analysis for all images can be found in the Supplementary information.

\section{Results and discussion}

Field-flow fractionation (FFF) is based on an open-channel technique for fractionation of molecular mixtures, which allows avoiding many problems in polymer analysis related to the stationary phase such as unspecific adsorption and extensive shear forces applied on large macromolecules. A FFF sub-technique, asymmetric flow field-flow fractionation (AF4) allows a fractionation of complex polymer systems according to molecular size by application of longitudinal and crossoriented flows in the channel (Kim et al., 2015; Malik \& Pasch, 2016; Shin, Hwang, Cho, \& Moon, 2007). The hyaluronan used in the study was characterized first using AF4 connected to MALS detectors (Wyatt, 
Table 1

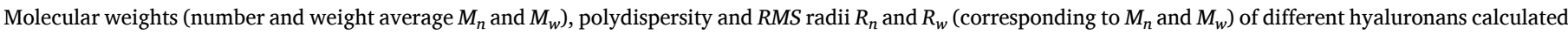
form AF4-MALS analysis.

\begin{tabular}{|c|c|c|c|c|c|c|}
\hline Sample & $M_{n}(\mathrm{kDa})$ & $M_{w}(\mathrm{kDa})$ & Polydispersity $\left(M_{w} / M_{n}\right)$ & $R_{n}(\mathrm{~nm})$ & $R_{w}(\mathrm{~nm})$ & Conformation plot slope \\
\hline HU190 & 161.8 & 186.2 & 1.151 & 33.2 & 37.3 & 0.49 \\
\hline HU470 & 377 & 473.3 & 1.255 & 58.2 & 63.2 & 0.23 \\
\hline HU720 & 668.2 & 723 & 1.082 & 93.4 & 98.7 & 0.60 \\
\hline HU1000 & 987.8 & 1006.5 & 1.019 & 128.1 & 130 & 0.58 \\
\hline
\end{tabular}

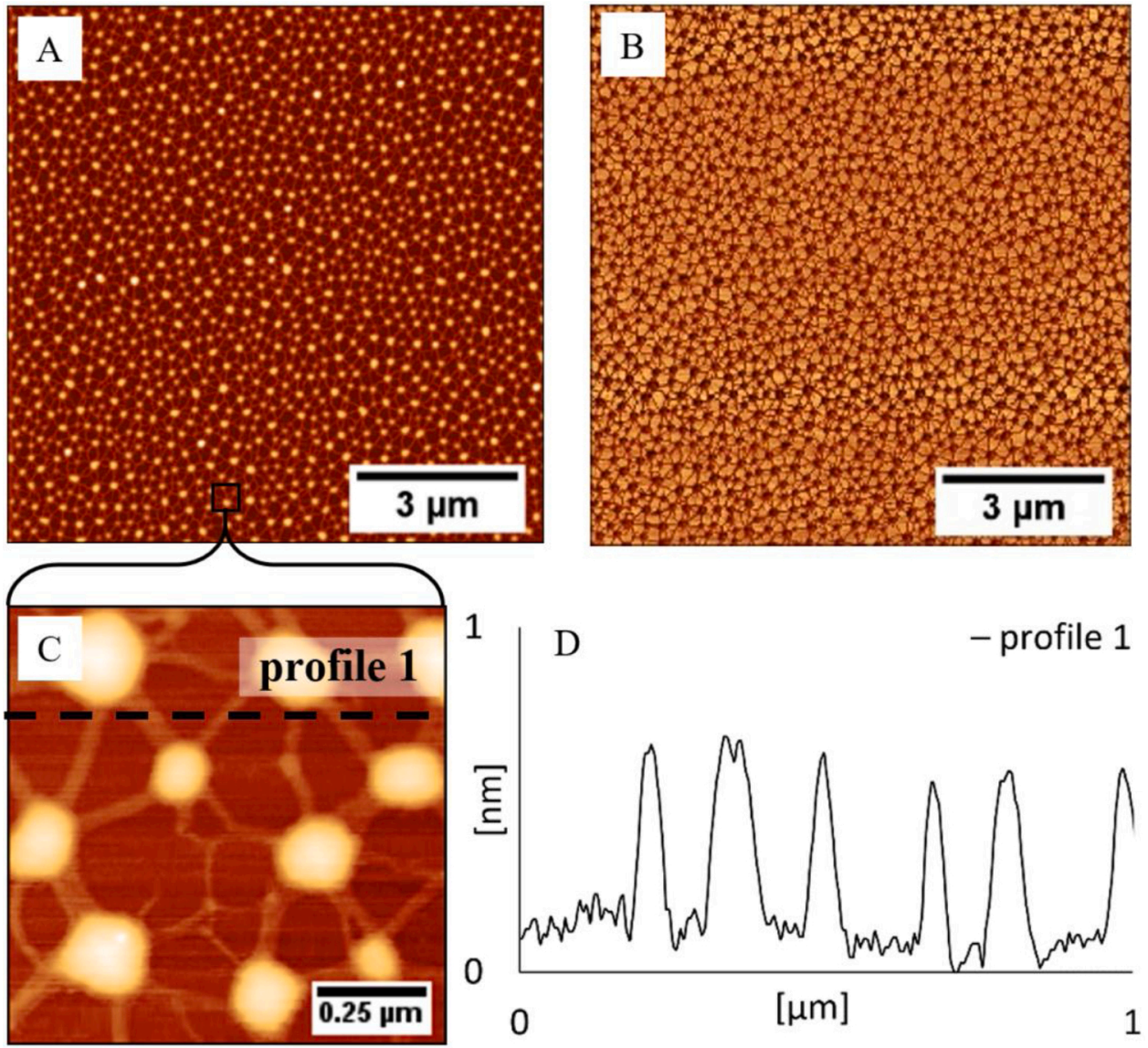

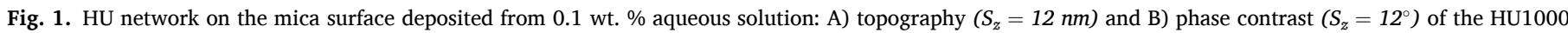

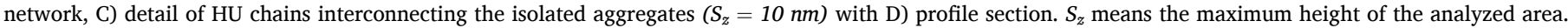

1997) in order to obtain molecular masses, polydispersity and shape factors of the polysaccharide chains in solutions (Table 1 ).

Evaluation of conformations slopes $(<0.33$ for sphere, $0.5-0.6$ for linear random coil, and $>1$ for rigid rod) revealed rather compact conformations in the used low ionic strength buffer systems.

AFM imaging of HU macromolecules has been presented in a number of studies demonstrating that their conformation is closely related to the properties of the substrate and the sample preparation methodology. While rinsing the excess solution from the carrier substrate with water leads to the attachment of the isolated chains on the surface by the process called "molecular combing", by omitting this step it is possible to obtain aggregated clusters of macromolecules interconnected by bundles of chains forming network (Cowman et al., 2005; Spagnoli et al., 2005). In the present study, we used this texture to present the connection between the solution history and the conformation of the solid-state polymer macromolecules. Using the AFM, the influence of different approaches to solution preparation was found not only on the character of the physical network reflecting the properties of the solution, but also the aggregation ability of the polymer on the surface of the carrier substrate.
Fig. 1 shows a topography image of a physical HU network (HU1000) deposited from a $0.1 \mathrm{wt}$ \% solution homogeneously covering the substrate surface (Fig. 1A) including phase contrast (Fig. 1B). In the detail in Fig. 1C, the fibers forming the network are shown. The fiber height of $0.75 \pm 0.04 \mathrm{~nm}$, seen from the profile section in Fig. 1D, corresponds to the diameter of the two polysaccharide chains of HU (Cowman et al., 2005).

\subsection{Concentration of deposited solution}

According to the literature, the intermediate polymeric network is formed in solution even at very low concentrations below 0.01 wt. \% (Spagnoli et al., 2005), where aggregate clusters are partly formed in solution and are completed when $\mathrm{HU}$ is deposited on the surface during drying (Cowman et al., 2005; Spagnoli et al., 2005). When diluting the initial 0.5 wt. \% solution before deposition on the surface, significant differences in the size and frequency of the aggregates are observed, as seen from the Fig. 2A (0.1 wt. \%) and 2B (0.01 wt. \%).

It is apparent from the profile sections next to the topography images (Fig. 2) that the height of the aggregates decreased approximately by 

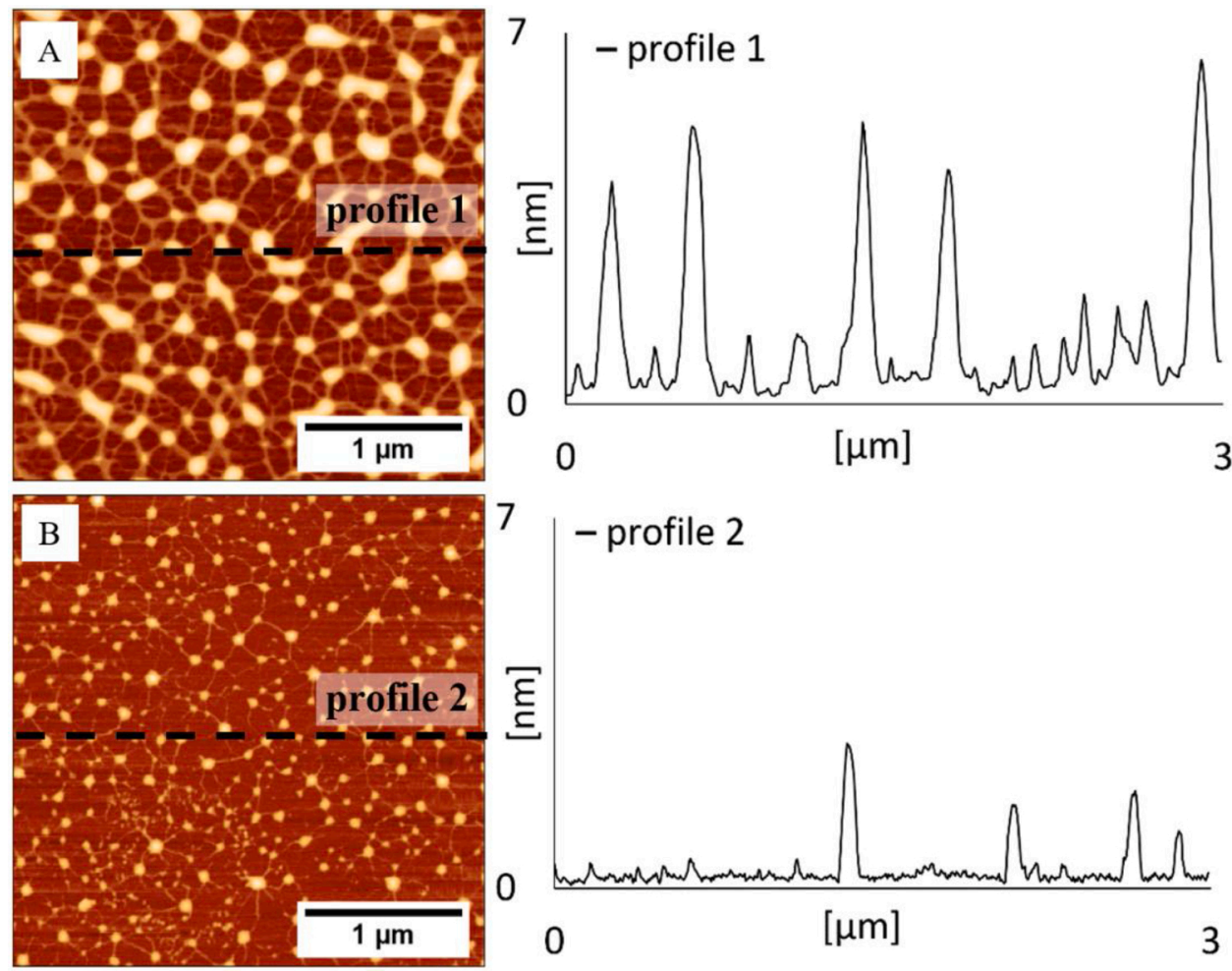

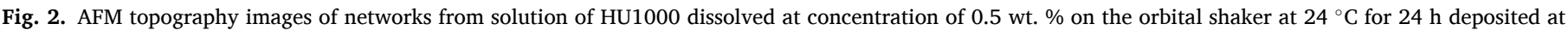

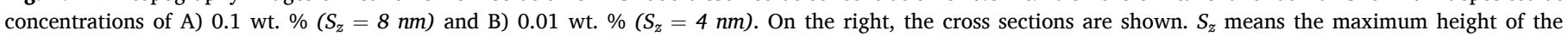
analyzed area.

half at a lower concentration. HU deposited at a concentration of $0.1 \mathrm{wt}$. $\%$ provides uniform surface coverage in one layer of the polymeric physical network. This observation corresponds to the knowledge of aggregation and netting concentration-dependence of $\mathrm{HU}$ in solution and on the phase interface and means that the selected immobilization and imaging of $\mathrm{HU}$ on the mica surface reflects directly the state of this macromolecular system in solution (Cowman et al., 2005; Matteini et al., 2009).

\subsection{Molecular weight}

The effect of the molecular weight of the deposited HU on the character of the network is presented in Fig. 3. As can be seen, HU190 with the molecular weight of $190 \mathrm{kDa}$ (Fig. 3A) shows a significantly lower mesh density compared to HU1000 (Fig. 3C). This phenomenon is consistent with the claim that long molecules form more nodes in solution than short molecules at the same concentration (Scott et al., 1991). For lower molecular weight HU, there is less opportunity for intermolecular interactions. For this reason, low $M_{w} \mathrm{HU}$ occur in solution as separate entities capable of forming organized domains more easily rearranged within individual polymer clusters. The free HU chains can then aggregate into larger clusters. The result is a decreasing number of aggregates and their enlargement.

\subsection{The shaking time during dissolving}

In case of dissolution of $\mathrm{HU}$ on the orbital shaker, one of the variables was the dissolution time. Solutions containing $0.1 \%$ HU720 were prepared on the orbital shaker for $24 \mathrm{~h}$ and $48 \mathrm{~h}$.

The results in Fig. 4 and the image analysis in Table 2 show that the longer mixing time results into doubled number of aggregates and increased number of crosslinks, while the coverage of the area with aggregates and nets remains similar. The results indicate that with prolonged influence of shear forces in the solution the shaking triggers disintegration of aggregates and higher crosslinking of the polymer chains.

\subsection{Storage}

The effect of storage on the formation of surface structures is presented in Fig. 5. HU1000 was dissolved at room temperature for $67 \mathrm{~h}$ on the shaking machine and then stored at quiescent condition at $4{ }^{\circ} \mathrm{C}$ for 2 - 4 days. Assuming that the HU chains are not degraded during the storage (Simulescu, Kalina, Mondek, \& Pekař, 2016), it is likely that the polymer network rearranged in the absence of shear forces since, intrachain interactions are favored over interchain ones. As a result, increasingly spatially distinct clusters are formed.

\subsection{Shear influence}

From the processing point of view, a method of preparation with different types of solution flow during HU dissolution was followed. Similar results were obtained by stirring the solution during dissolving by orbital shaker at $25{ }^{\circ} \mathrm{C}$ and magnetic stirrer at $45{ }^{\circ} \mathrm{C}$ as shown in Fig. 6A and B, respectively. However, a significant difference was noted in case of dissolving $\mathrm{HU}$ in the temperature field flow chamber (TFFC, 

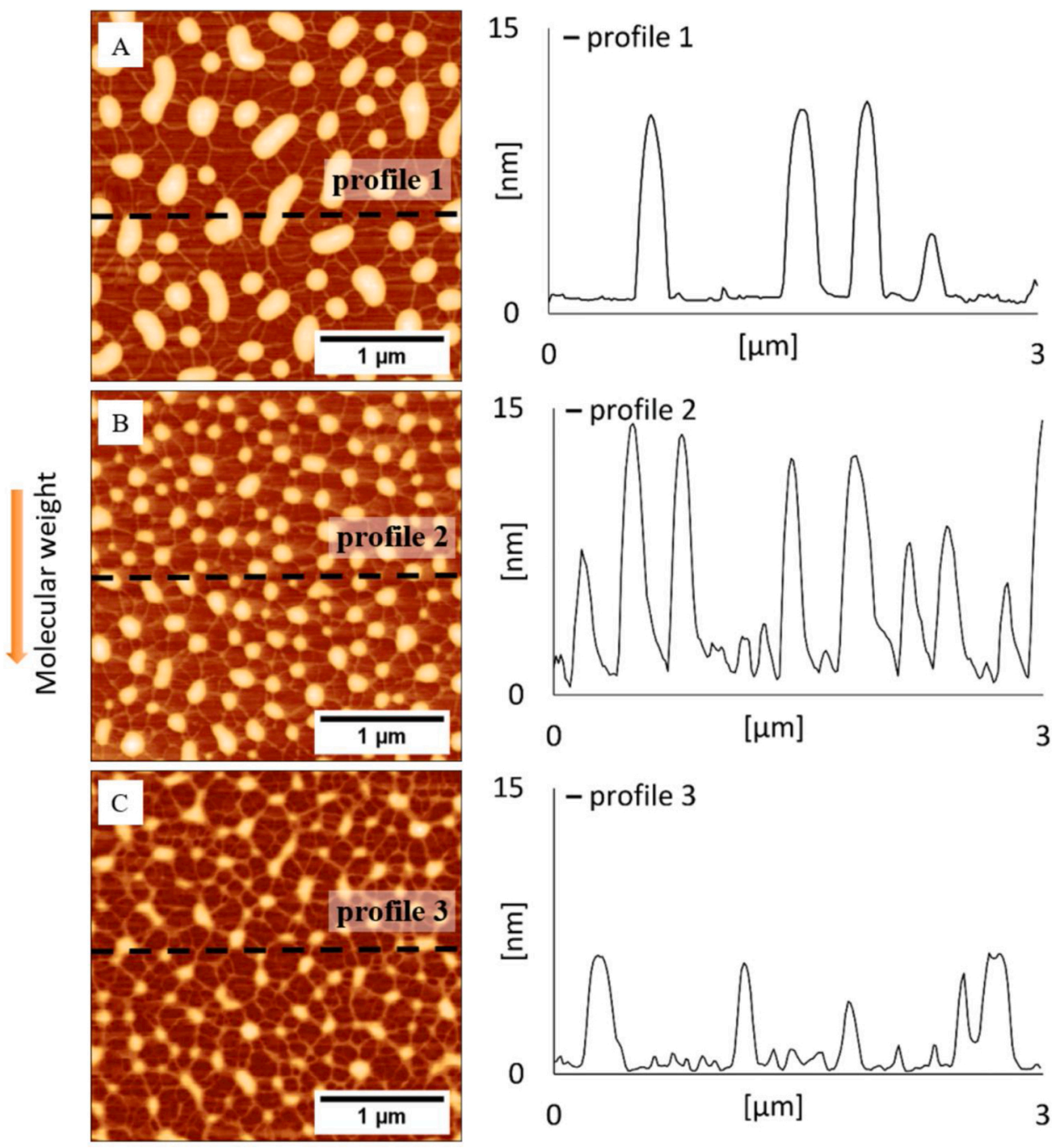

Fig. 3. AFM topography images of networks from HU solutions A) HU190 $\left(S_{z}=13 \mathrm{~nm}\right)$ B) HU470 $\left(S_{z}=17 \mathrm{~nm}\right)$ and C) HU1000 ( $\left.S_{z}=14 \mathrm{~nm}\right)$ with starting concentration $0.5 \mathrm{wt} . \%$ deposited in concentration $0.1 \mathrm{wt}$. \% dissolved in $24 \mathrm{~h}$ at $24{ }^{\circ} \mathrm{C}$ on the shaking machine. $S_{z}$ means the maximum height of the analyzed area. On the right, the cross sections are shown.

Fig. 6C). In TFFC, temperature gradients $\left(50 / 20^{\circ} \mathrm{C}\right.$, middle temperature in solution $T_{s}=45^{\circ} \mathrm{C}$ ) influence a rearrangement of macromolecules in solution due to generated Bénard-Marangoni convection, as described in our previous work (Minařík et al., 2017). The self-organized fluid flows associated with intense shear stress of macromolecular chains that occurs in the solution during dissolution affects the frequency of interchain interactions and causes a higher cross-linking density, which is evident from the higher frequency of aggregates, or nodes, in the polymer network on the surface. Interestingly, the convective flows in the solution layer triggered multiple number of polymer bundles connecting the individual aggregates.

Despite the slightly higher number of aggregates in the case of TFFC, due to their lower diameter, the area coverage with aggregates for all approaches was $\sim 20 \%$. However, the surface coverage by the fibrillar networks differs significantly. In the case of TFFC, the net coverage is almost twice as high as with magnetic stirrer, see Table 3.

\subsection{HU at physiological condition}

In addition to the physical influences on the aggregation and networking of the $\mathrm{HU}$ molecules during the solubilization, the influence of increased ionic strength close to physiological conditions was tested. Fig. 7 shows the difference between the structure of HU deposited form water (Fig. 7A) and isotonic $0.9 \%$ sodium chloride (Fig. 7B). In the physiological solution, a network structure is formed which is initiated by the formation of hydrogen bridges between the chains, the concentration of ions in the solution has a secondary role in this case (Wu, Ai, Chen, Kang, \& Cui, 2013). However, larger aggregates of HU on the surface are formed in the presence of saline at the expense of mesh density. In general, the presence of salts promotes a more collapsed $\mathrm{HU}$ conformation. We assume that the interaction of these primary clusters leads to the formation of larger aggregates, which corresponds to previous observations relate to low $M_{w} \mathrm{HU}$ and long-term storage.

Polysaccharide properties in solutions are determined mainly by the inter- and intra-chain interactions via hydrogen bonds, hydrophobic interactions and, in the case of polyelectrolytes, by electrostatic 

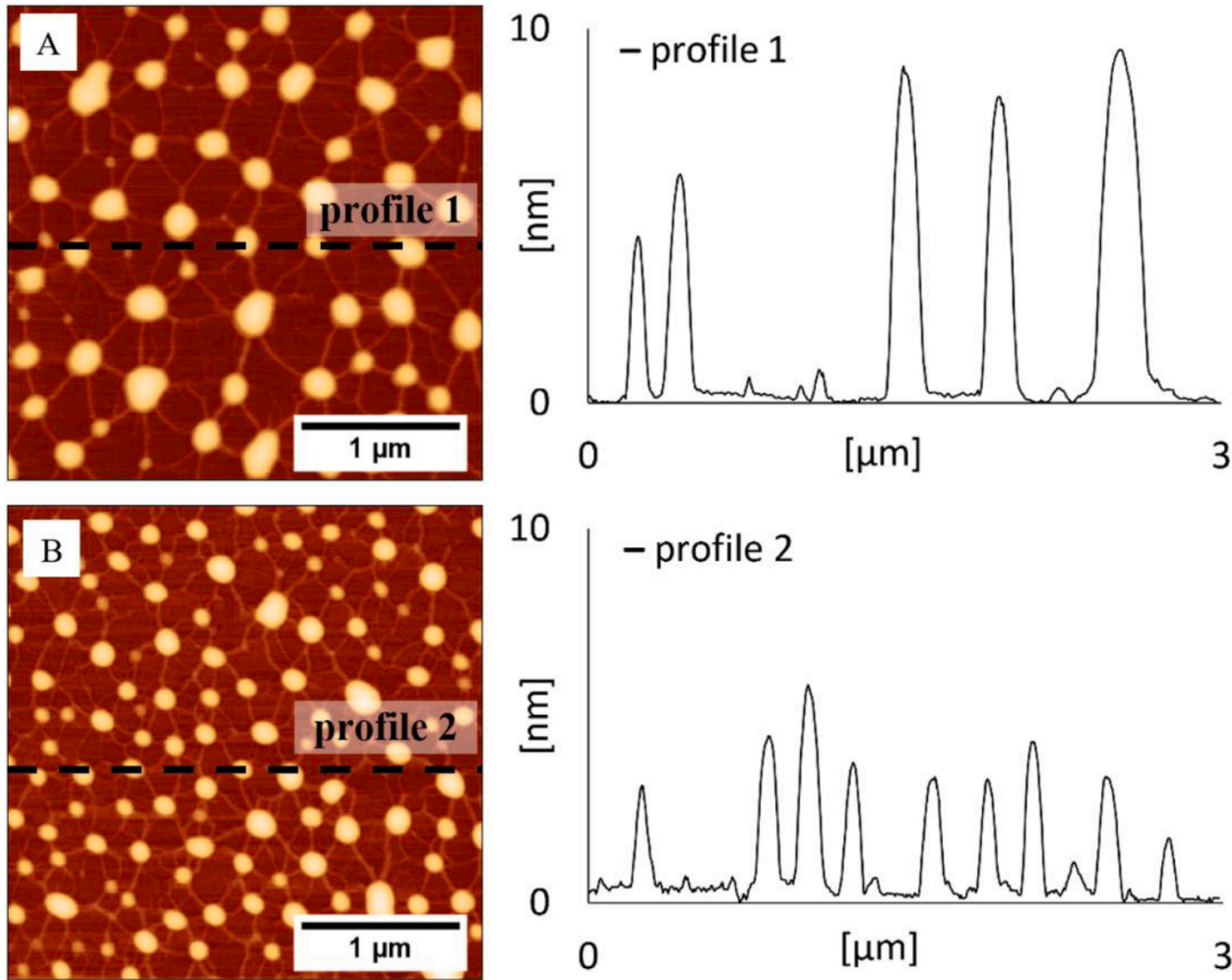

Fig. 4. AFM topography images of networks from solution of HU720 deposited in concentration $0.1 \mathrm{wt} . \%$ dissolved on the shaker at $24{ }^{\circ} \mathrm{C}$ for A) $24 \mathrm{~h}\left(S_{z}=17 \mathrm{~nm}\right)$ and B) $48 \mathrm{~h}\left(S_{z}=11 \mathrm{~nm}\right) . S_{z}$ means the maximum height of the analyzed area. On the right, the cross sections are shown.

Table 2

Comparison of network structures from solutions with different dissolving time, analyzed area $1 \mu \mathrm{m}^{2}$.

\begin{tabular}{|c|c|c|c|c|c|c|c|}
\hline $\begin{array}{l}\text { Dissolution time } \\
{[\mathrm{h}]}\end{array}$ & $\begin{array}{l}\text { Aggregates } \\
\text { number }\end{array}$ & $\begin{array}{l}\text { Maximum height } \\
\text { [nm] }\end{array}$ & $\begin{array}{l}\text { Mean height } \\
{[\mathrm{nm}]}\end{array}$ & $\begin{array}{l}\text { Average projected area } \\
{\left[10^{3} \mathrm{~nm}^{2}\right]}\end{array}$ & $\begin{array}{l}\text { Aggregates covering } \\
{[\%]}\end{array}$ & $\begin{array}{l}\text { Network covering } \\
{[\%]}\end{array}$ & $\begin{array}{l}\text { Total covering } \\
{[\%]}\end{array}$ \\
\hline 24 & $9.8 \pm 1.2$ & $12.1 \pm 1.2$ & $5.2 \pm 0.3$ & $22 \pm 2$ & $18.0 \pm 0.6$ & $12.0 \pm 2.0$ & $29.9 \pm 1.5$ \\
\hline 48 & $17.0 \pm 0.8$ & $8.2 \pm 0.4$ & $3.9 \pm 0.1$ & $9 \pm 1$ & $15.5 \pm 0.7$ & $15.7 \pm 1.7$ & $31.2 \pm 1.4$ \\
\hline
\end{tabular}

interactions. In addition to the intrinsic character of the polymeric chain, a number of extrinsic variables have to be considered in processing of polysacharide-based systems, Thus, temperature, ionic strength and $\mathrm{pH}$ play also important roles in the conformation changes (Rinaudo, 2006). Further, the pH of the environment also affects the adhesion of HU to the surfaces (Choi et al., 2015), as well as the charge and the chemical composition of the surface (Spagnoli et al., 2005). Specific changes in mesh structure initiated by molecular weight of $\mathrm{HU}$ correspond to the well-known HU- $M_{w}$ dependence, when the intermolecular interactions between HA chains are favoured by chain length (Shen et al., 2011). This phenomenon can have a signifficant effect on the bioactivity in the preparation of biomaterial surfaces based on hyaluronan (Morra, 2005).

\section{Conclusion}

In this study, the network-forming and aggregation properties of $\mathrm{HU}$ on the mica surface have been examined using AFM. HU, dissolved at various temperatures and time regimes, were deposited on the charged mica surface and the network structures formed on the liquid-solid interface were imaged in the tapping mode.

The research confirmed the effect of the average molecular weight and the concentration of $\mathrm{HU}$ in solution on the aggregation and networkforming properties. In agreement with previous observations, the increase in the mesh density with increasing molecular weight was observed. Furthermore, it has been found that the tendency for crosslinking and aggregation varies during storage of the solution and also depends significantly on its treatment during dissolution, particularly on the temperature and the flow character. The time for which the HU solution is exposed to the dissolution conditions also plays an important role. The observations shows that during storage of water solutions at low temperature, the polymer clusters reorganize and form larger units, similar to the influence of ions present in solution. This rearrangement also occurs spontaneously in low $M_{w}$ HU water solutions. The opposite phenomenon can be observed in case of gentle shear stress of polymer coils in solution.

These findings point to the significant temperature and time dependence of the network-forming and aggregation abilities of $\mathrm{HU}$ proving the influence of ambient conditions on the ratio between interchain and intra-chain interactions. This knowledge is contributive for progress in understanding the conformational stability of hyaluronan in aqueous solutions. Furthermore, presented preparation approaches, especially dissolution $\mathrm{HU}$ in temperature gradient, provide a possible way to modify the network texture of HU. Our findings show that in 

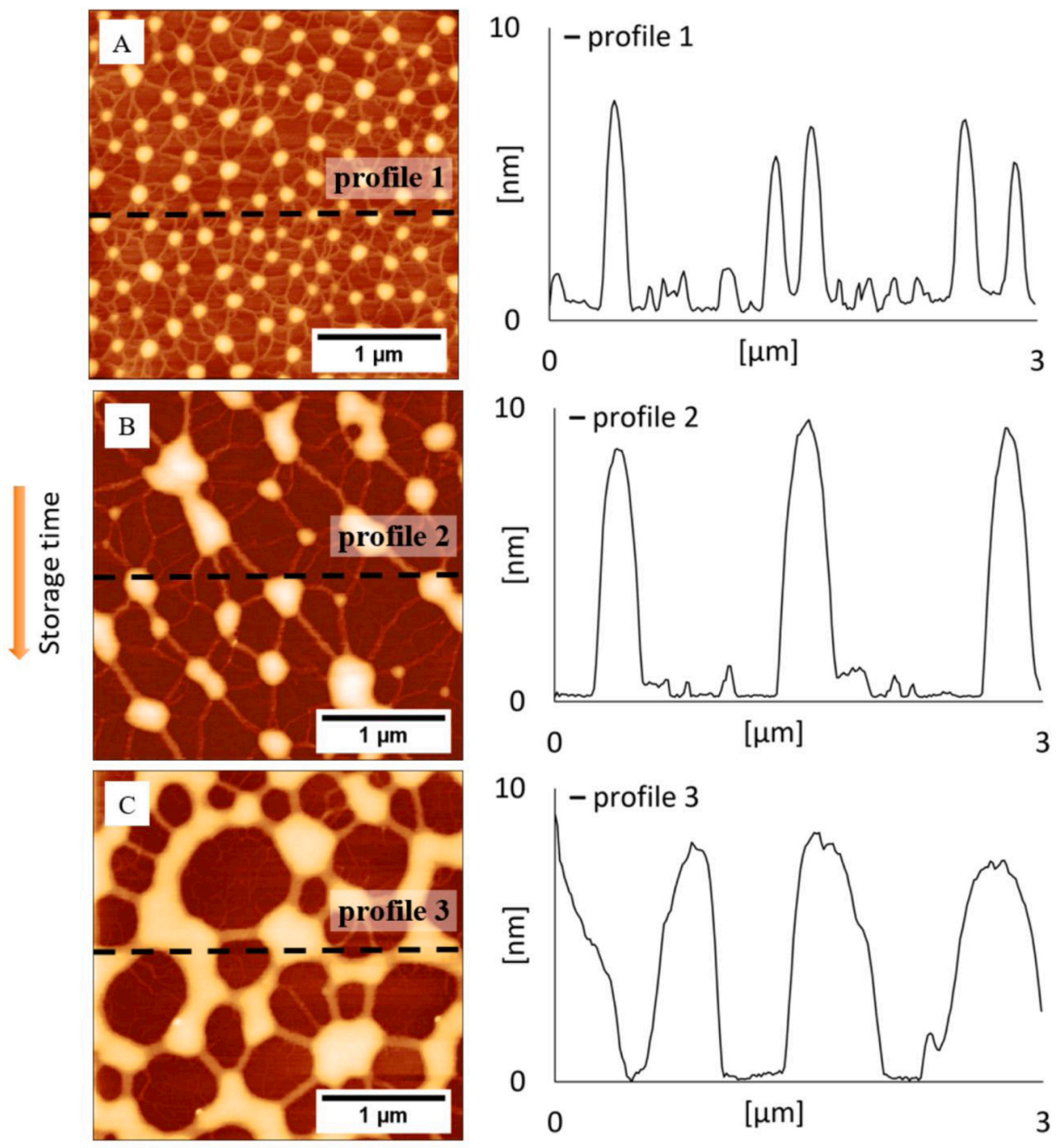

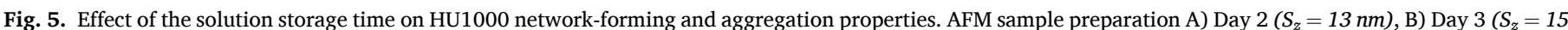

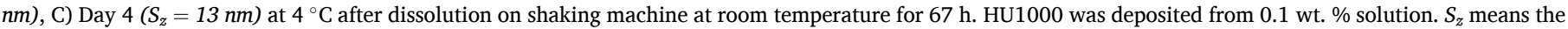
maximum height of the analyzed area. On the right, the cross sections are shown. 
A) Orbital shaker

low temperature / low shear stress $T_{1}=T_{2}=T_{s}$

\begin{tabular}{|c|}
\hline$T_{1}=25^{\circ} \mathrm{C}$ \\
\hline$T_{S}=25^{\circ} \mathrm{C} \longleftrightarrow$ \\
\hline$T_{2}=25^{\circ} \mathrm{C}$ \\
\hline
\end{tabular}
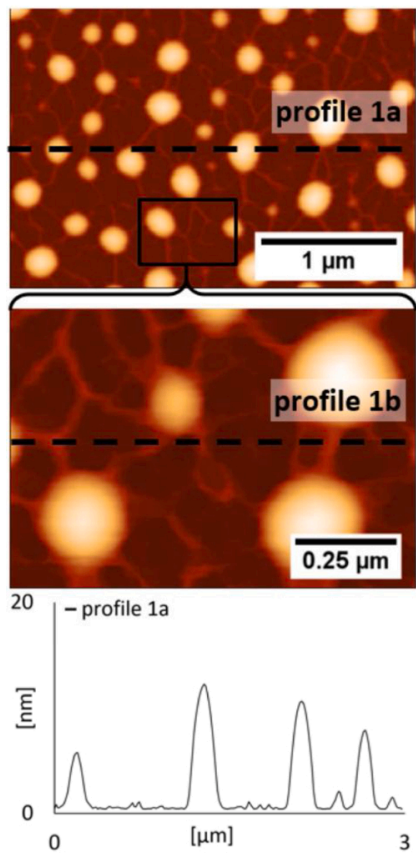

- profile $1 b$

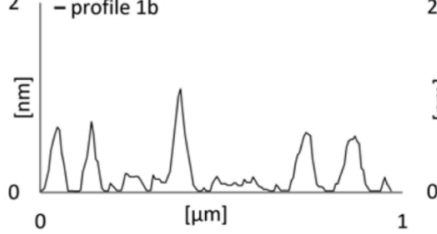

B) Magnetic stirrer

high temperature / medium shear stress high temperature / high shear stress
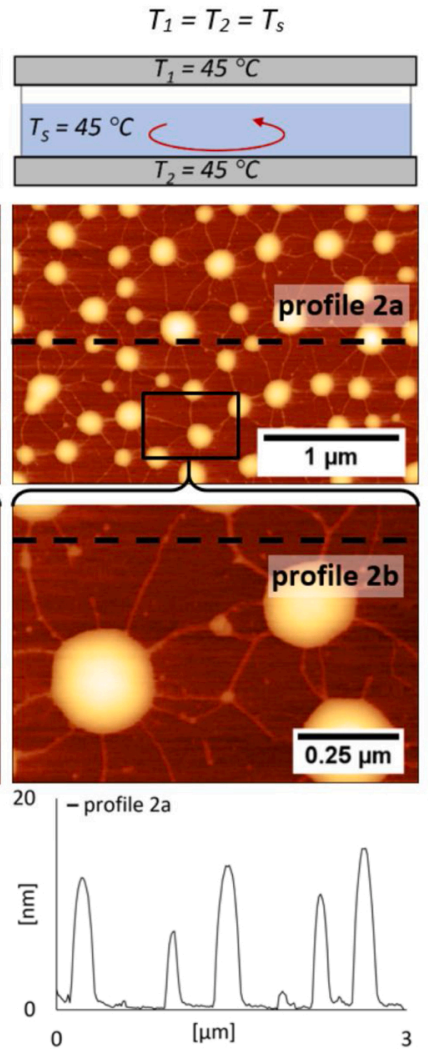

C) TFFC device
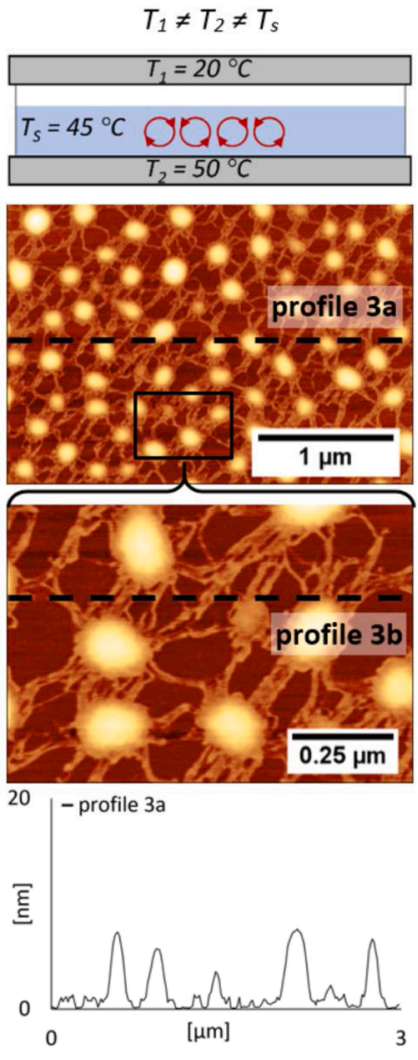
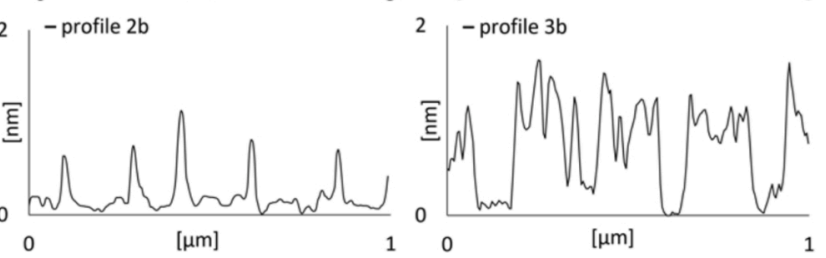

Fig. 6. AFM topography images of networks from solution of HU470 solubilized at concentration of $0.5 \mathrm{wt}$ \% and deposited at a concentration of 0.1 wt. \%. A) solubilization on orbital shaker at $25^{\circ} \mathrm{C}$ for $24 \mathrm{~h}\left(S_{z}=16 \mathrm{~nm}\right)$, B) solubilization on magnetic stirrer at $45{ }^{\circ} \mathrm{C}$ for $24 \mathrm{~h}\left(S_{z}=21 \mathrm{~nm}\right)$ and C) solubilization in TFFC at temperature gradient from 20 to $50{ }^{\circ} \mathrm{C}, T_{\mathrm{s}}=$ $45{ }^{\circ} \mathrm{C}\left(S_{z}=12 \mathrm{~nm}\right) . S_{z}$ means the maximum height of the analyzed area. Cross sections along the dashed lines are shown below the respective AFM images.

Table 3

Attributes of HU networks after deposition of the solutions from different dissolving processes, analyzed area $1 \mu \mathrm{m}^{2}$.

\begin{tabular}{|c|c|c|c|c|c|c|c|}
\hline $\begin{array}{l}\text { Dissolution } \\
\text { process }\end{array}$ & $\begin{array}{l}\text { Aggregates } \\
\text { number }\end{array}$ & $\begin{array}{l}\text { Maximum height } \\
{[\mathrm{nm}]}\end{array}$ & $\begin{array}{l}\text { Mean height } \\
{[\mathrm{nm}]}\end{array}$ & $\begin{array}{l}\text { Average projected area } \\
{\left[10^{3} \mathrm{~nm}^{2}\right]}\end{array}$ & $\begin{array}{l}\text { Aggregates covering } \\
{[\%]}\end{array}$ & $\begin{array}{l}\text { Network covering } \\
{[\%]}\end{array}$ & $\begin{array}{l}\text { Total covering } \\
{[\%]}\end{array}$ \\
\hline Orbital shaker & $8.6 \pm 0.7$ & $13.6 \pm 0.6$ & $5.5 \pm 0.2$ & $22 \pm 1$ & $18.3 \pm 1.2$ & $25.5 \pm 1.0$ & $43.8 \pm 1.6$ \\
\hline Magnetic stirrer & $11.6 \pm 0.8$ & $18.7 \pm 0.5$ & $8.1 \pm 0.3$ & $18 \pm 2$ & $19.5 \pm 0.3$ & $12.6 \pm 0.6$ & $32.0 \pm 0.5$ \\
\hline TFFC device & $13.8 \pm 0.7$ & $9.4 \pm 0.5$ & $4.6 \pm 0.2$ & $12 \pm 1$ & $19.3 \pm 0.6$ & $39.0 \pm 3.0$ & $58.0 \pm 3.0$ \\
\hline
\end{tabular}

addition to above mentioned physico-chemical influences, the type of applied shear forces generated by the different type of mixing such as orbital, overhead or convectional dissolution process affect the aggregation and crosslinking of HU. In comparison to conventional mixing, apparently increased shear stress in case of the Bénard-Marangoni convection generated by the temperature gradient, enables significant unfolding of the HU chains and inter-crosslinking of the HU network. These finding points towards importance of the shear forces applied during the processing of $\mathrm{HU}$ solutions. Systematic studies on the $\mathrm{HU}$ solution rheology and its effects on the morphology of the HU network during deposition to investigate systematically the effects of solution viscosity and shear stress are underway to find out new ways for hyaluronan crosslinking in the absence of chemical agents, which is highly desirable in the preparation of hydrogels (Trombino, Servidio, Curcio, \& Cassano, 2019) with shear thing behavior suitable for an 3D-printing deposition.

\section{CRediT authorship contribution statement}

Karolína Kocourková: Investigation, Formal analysis, Visualization, Writing - original draft. Lenka Musilová: Formal analysis. Petr Smolka: Writing - review \& editing. Aleš Mráček: Project administration, Resources. Martin Humenik: Investigation, Formal analysis, Writing - review \& editing. Antonín Minařík: Conceptualization, Methodology, Supervision, Writing - review \& editing. 

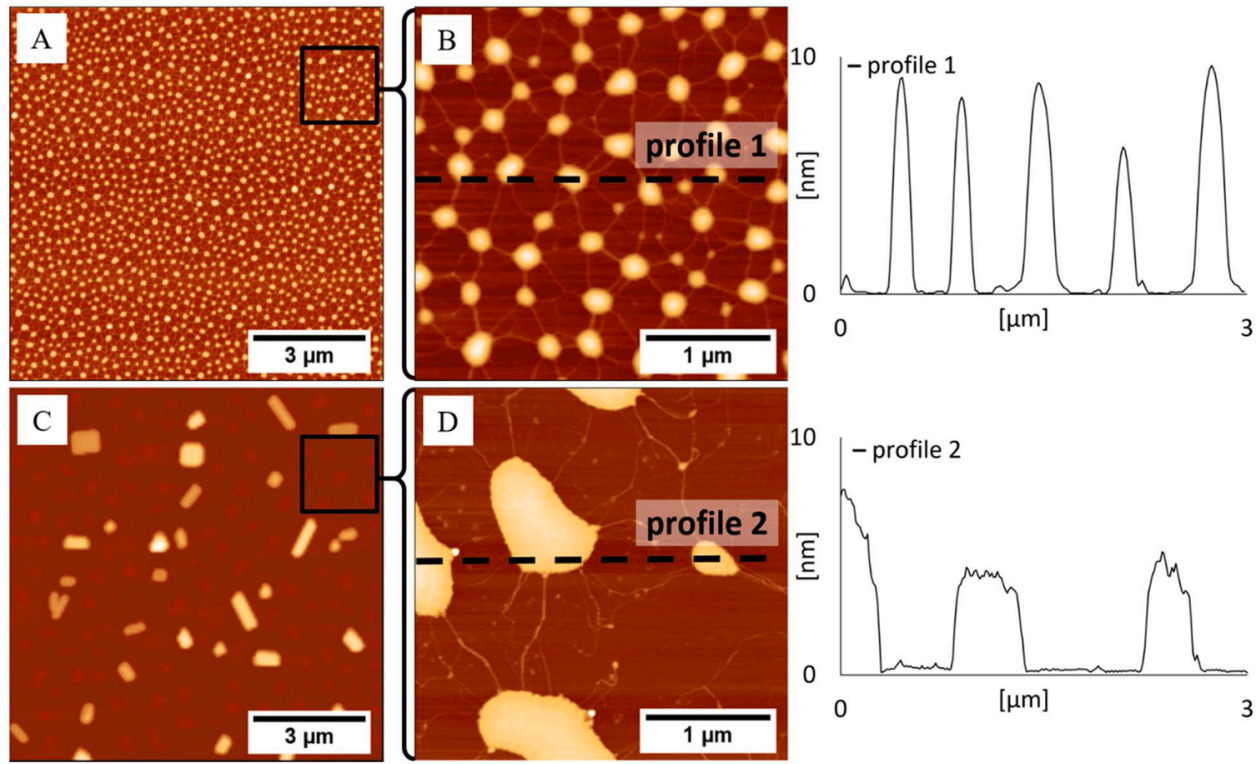

Fig. 7. AFM topography images of networks deposited from solution of HU720 in water: A) surface area $10 \times 10 \mu \mathrm{m}^{2}$ and B) surface area 3 $\times 3 \mu \mathrm{m}^{2}, \mathrm{Sz}=13 \mathrm{~nm}$ for both, and in saline: C) surface with $\mathrm{NaCl}$ crystals and $\mathrm{HU}$, area $10 \times 10$ $\mu \mathrm{m}^{2}, S_{z}=400 \mathrm{~nm}$ and D) HU aggregates and chains, surface area $3 \times 3 \mu \mathrm{m}^{2}, S_{z}=12 \mathrm{~nm}$. HU was dissolved at $0.5 \mathrm{wt}$ \% at room temperature on orbital shaker for $24 \mathrm{~h}$ and deposited at concentration of 0.1 wt. \%. $S_{z}$ means the maximum height of the analyzed area. On the right, the cross sections along the dashed lines are shown.

\section{Declaration of Competing Interest}

The authors report no declarations of interest.

\section{Acknowledgments}

This work was supported by the Ministry of Education, Youth and Sports of the Czech Republic, Bayerisch-Tschechische Hochschulagentur BTHA/BAYHOST (project LTAB19019), the Ministry of Education, Youth and Sports of the Czech Republic DKRVO (RP/CPS/2020/003) and by TBU grant No. IGA/FT/2020/005 funded from the resources of specific university research. We would like to thank Prof. Dr. Thomas Scheibel (Department of Biomaterials, Bayreuth) for enabling us to use AF4-MALS equipment.

\section{Appendix A. Supplementary data}

Supplementary material related to this article can be found, in the online version, at doi:https://doi.org/10.1016/j.carbpol.2020.117307.

\section{References}

Almond, A., DeAngelis, P., \& blundell, C. (2006). Hyaluronan: The local solution conformation determined by NMR and computer modeling is close to a contracted left-handed 4-fold helix. Journal of Molecular Biology, 358(5), 1256-1269.

Bishop, P. (2000). Structural macromolecules and supramolecular organisation of the vitreous gel. Progress in Retinal and Eye Research, 19(3), 323-344.

Choi, J., Kim, S., Linardy, E., Dreaden, E., Zhdanov, V., Hammond, P., et al. (2015). Adsorption of hyaluronic acid on solid supports: Role of $\mathrm{pH}$ and surface chemistry in thin film self-assembly. Journal of Colloid and Interface Science, 448, 197-207.

Cowman, M., \& Matsuoka, S. (2005). Experimental approaches to hyaluronan structure Carbohydrate Research, 340(5), 791-809.

Cowman, M., Hittner, D., \& Feder-Davis, J. (1996). 13 C-NMR studies of hyaluronan: Conformational sensitivity to varied environments. Macromolecules, 29(8), 2894-2902.

Cowman, M., Li, M., \& Balazs, E. (1998). Tapping mode atomic force microscopy of hyaluronan: Extended and intramolecularly interacting chains. Biophysical Journal, 75(4), 2030-2037.

Cowman, M., Spagnoli, C., Kudasheva, D., Li, M., Dyal, A., Kanai, S., et al. (2005). Extended, relaxed, and condensed conformations of hyaluronan observed by atomic force microscopy. Biophysical Journal, 88(1), 590-602.

Day, A., \& Sheehan, J. (2001). Hyaluronan: Polysaccharide chaos to protein organisation. Current Opinion in Structural Biology, 11(5), 617-622.

Deng, X., Attalla, R., Sadowski, L., Chen, M., Majcher, M., Urosev, I., et al. (2017). Autonomously self-adhesive hydrogels as building blocks for additive manufacturing. Biomacromolecules, 19(1), 62-70.

Dicker, K., Gurski, L., Pradhan-Bhatt, S., Witt, R., Farach-Carson, M., \& Jia, X. (2014). Hyaluronan: A simple polysaccharide with diverse biological functions. Acta Biomaterialia, 10(4), 1558-1570.
Dosio, F., Arpicco, S., Stella, B., \& Fattal, E. (2016). Hyaluronic acid for anticancer drug and nucleic acid delivery. Advanced Drug Delivery Reviews, 97, 204-236.

Evanko, S., Parks, W., \& Wight, T. (2016). Intracellular hyaluronan in arterial smooth muscle cells: Association with microtubules, RHAMM, and the mitotic spindle. Journal of Histochemistry and Cytochemistry, 52(12), 1525-1535.

Fallacara, A., Baldini, E., Manfredini, S., \& Vertuani, S. (2018). Hyaluronic acid in the third millennium. Polymers, 10(7), 1-36.

Foglarová, M., Chmelař, J., Huerta-Angeles, G., Vágnerová, H., Kulhánek, J., Barton̆ Tománková, K., et al. (2016). Water-insoluble thin films from palmitoyl hyaluronan with tunable properties. Carbohydrate Polymers, 144, 68-75.

Giannotti, M., Rinaudo, M., \& Vancso, G. (2007). Force spectroscopy of hyaluronan by atomic force microscopy: From hydrogen-bonded networks toward single-chain behavior. Biomacromolecules, 8(9), 2648-2652.

Giubertoni, G., Burla, F., Martinez-Torres, C., Dutta, B., Pletikapic, G., Pelan, E., et al. (2019). Molecular origin of the elastic state of aqueous hyaluronic acid. The Journal of Physical Chemistry B, 123(14), 3043-3049.

Gribbon, P., Heng, B., \& Hardingham, T. (1999). The molecular basis of the solution properties of hyaluronan investigated by confocal fluorescence recovery after photobleaching. Biophysical Journal, 77(4), 2210-2216.

Grossutti, M., \& Dutcher, J. (2016). Correlation between chain architecture and hydration water structure in polysaccharides. Biomacromolecules, 17(3), 1198-1204.

Gřundělová, L., Mráček, A., Kašpárková, V., Minařík, A., \& Smolka, P. (2013). The influence of quarternary salt on hyaluronan conformation and particle size in solution. Carbohydrate Polymers, 98(1), 1039-1044.

Gunning, A., Morris, V., Al-Assaf, S., \& Phillips, G. (1996). Atomic force microscopic studies of hylan and hyaluronan. Carbohydrate Polymers, 30(1), 1-8.

Hargittai, I., \& Hargittai, M. (2008). Molecular structure of hyaluronan: An introduction. Structural Chemistry, 19(5), 697-717.

Heinegård, D. (2009). Fell-Muir Lecture: Proteoglycans and more - from molecules to biology. International Journal of Experimental Pathology, 90(6), 575-586.

Highley, C., Prestwich, G., \& Burdick, J. (2016). Recent advances in hyaluronic acid hydrogels for biomedical applications. Current Opinion in Biotechnology, 40, 35-40.

Jacoboni, I., Valdrè, U., Mori, G., Quaglino, D., \& Pasquali-Ronchetti, I. (1999). Hyaluronic acid by atomic force microscopy. Journal of Structural Biology, 126(1), $52-58$.

Kim, B., Woo, S., Park, Y., Hwang, E., \& Moon, M. (2015). Ionic strength effect on molecular structure of hyaluronic acid investigated by flow field-flow fractionation and multiangle light scattering. Analytical and Bioanalytical Chemistry, 407(5), $1327-1334$.

Kutálková, E., Hrnčiř́k, J., Witasek, R., \& Ingr, M. (2020). Effect of solvent and ions on the structure and dynamics of a hyaluronan molecule. Carbohydrate Polymers, 234, $1-8$.

Lapčík, L., Lapčík, L., De Smedt, S., Demeester, J., \& Chabreček, P. (1998). Hyaluronan: Preparation, structure, properties, and applications. Chemical Reviews, 98(8), 2663-2684.

Malik, M., \& Pasch, H. (2016). Field-flow fractionation: New and exciting perspectives in polymer analysis. Progress in Polymer Science, 63, 42-85.

Matteini, P., Dei, L., Carretti, E., Volpi, N., Goti, A., \& Pini, R. (2009). Structural behavior of highly concentrated hyaluronan. Biomacromolecules, 10(6), 1516-1522.

Minařík, A., Smolka, P., Minařík, M., Mráček, A., Rajnohová, E., Minaříková, M., et al. (2017). A special instrument for the defined modification of polymer properties in solutions and polymer layers. Measurement, 97, 218-225.

Mondschein, R., Kanitkar, A., Williams, C., Verbridge, S., \& Long, T. (2017). Polymer structure-property requirements for stereolithographic 3D printing of soft tissue engineering scaffolds. Biomaterials, 140, 170-188. 
Morra, M. (2005). Engineering of biomaterials surfaces by hyaluronan. Biomacromolecules, 6(3), 1205-1223.

Mráček, A., Gřundělová, L., Minařík, A., Veríssimo, L., Barros, M., \& Ribeiro, A. (2015) Characterization at $25^{\circ} \mathrm{C}$ of sodium hyaluronate in aqueous solutions obtained by transport techniques. Molecules, 20(4), 5812-5824.

Musilová, L., Kašpárková, V., Mráček, A., Minařík, A., \& Minařík, M. (2019). The behaviour of hyaluronan solutions in the presence of Hofmeister ions: A light scattering, viscometry and surface tension study. Carbohydrate Polymers, 212, 395-402.

Nestor, G., \& Sandström, C. (2017). NMR study of hydroxy and amide protons in hyaluronan polymers. Carbohydrate Polymers, 157, 920-928.

Nimmo, C., Owen, S., \& Shoichet, M. (2011). Diels-alder click cross-linked hyaluronic acid hydrogels for tissue engineering. Biomacromolecules, 12(3), 824-830.

Rinaudo, M. (2006). Non-covalent interactions in polysaccharide systems. Macromolecular Bioscience, 6(8), 590-610.

Scott, J. (1992). Supramolecular organization of extracellular-matrix glycosaminoglycans, invitro and in the tissues. The FASEB Journal, 6(9), 2639-2645.

Scott, J., \& Heatley, F. (1999). Hyaluronan forms specific stable tertiary structures in aqueous solution: A 13C NMR study. Proceedings of the National Academy of Sciences, 96(9), 4850-4855.

Scott, J., Cummings, C., Brass, A., \& Chen, Y. (1991). Secondary and tertiary structures of hyaluronan in aqueous solution, investigated by rotary shadowing-electron microscopy and computer simulation. Hyaluronan is a very efficient networkforming polymer. The Biochemical Journal, 274(3), 699-705.

Shanmuga Doss, S., Bhatt, N., \& Jayaraman, G. (2017). Improving the accuracy of hyaluronic acid molecular weight estimation by conventional size exclusion chromatography. Journal of Chromatography B, 1060, 255-261.

Shen, L., Chaudouet, P., Ji, J., \& Picart, C. (2011). pH-amplified multilayer films based on hyaluronan: Influence of HA molecular weight and concentration on film growth and stability. Biomacromolecules, 12(4), 1322-1331.
Shin, D., Hwang, E., Cho, I., \& Moon, M. (2007). Molecular weight and structure characterization of sodium hyaluronate and its gamma radiation degradation products by flow field-flow fractionation and on-line multiangle light scattering. Journal of Chromatography A, 1160(1-2), 270-275.

Simulescu, V., Kalina, M., Mondek, J., \& Pekař, M. (2016). Long-term degradation study of hyaluronic acid in aqueous solutions without protection against microorganisms. Carbohydrate Polymers, 137, 664-668.

Spagnoli, C., Korniakov, A., Ulman, A., Balazs, E., Lyubchenko, Y., \& Cowman, M. (2005). Hyaluronan conformations on surfaces: Effect of surface charge and hydrophobicity. Carbohydrate Research, 340(5), 929-941.

Trombino, S., Servidio, C., Curcio, F., \& Cassano, R. (2019). Strategies for hyaluronic acid-based hydrogel design in drug delivery. Pharmaceutics, 11(8), 1-17.

Van Vlierberghe, S., Dubruel, P., \& Schacht, E. (2011). Biopolymer-based hydrogels as scaffolds for tissue engineering applications: A review. Biomacromolecules, 12(5), 1387-1408.

Wieland, D., Degen, P., Zander, T., Gayer, S., Raj, A., An, J., et al. (2016). Structure of DPPC-hyaluronan interfacial layers - Effects of molecular weight and ion composition. Soft Matter, 12(3), 729-740.

Wu, S., Ai, L., Chen, J., Kang, J., \& Cui, S. (2013). Study of the mechanism of formation of hyaluronan putty at pH 2.5: Part I. Experimental measurements. Carbohydrate Polymers, 98(2), 1677-1682.

Wyatt, P. (1997). Multiangle light scattering: The basic tool for macromolecular characterization. Instrumentation Science \& Technology, 25(1), 1-18.

Xu, X., Jha, A., Harrington, D., Farach-Carson, M., \& Jia, X. (2012). Hyaluronic acidbased hydrogels: From a natural polysaccharide to complex networks. Soft Matter, 8 (12), 1-28.

Zellermann, A., Bergmann, D., \& Mayer, C. (2013). Cation induced conformation changes in hyaluronate solution. European Polymer Journal, 49(1), 70-79.

Zheng, Y., Yang, J., Liang, J., Xu, X., Cui, W., Deng, L., et al. (2019). Bioinspired hyaluronic acid/phosphorylcholine polymer with enhanced lubrication and antiinflammation. Biomacromolecules, 20(11), 4135-4142. 